\title{
Risk of developing CMV retinitis following non-ocular CMV end organ disease in AIDS patients
}

Frank D Verbraak, Gerardus J van den Horn, Jan T M van der Meer, Darius Paydafar, Fibo J W ten Kate, Pauline M E Wertheim-van Dillen, Aize Kijlstra

Department of Ophthalmology, Academic Medical Centre, University of Amsterdam, Amsterdam, Netherlands F D Verbraak G J van den Horn A Kijlstra

Department of Internal Medicine, Division of Infectious diseases, Tropical medicine, and AIDS, Academic Medical Centre, University of Amsterdam, Amsterdam, Netherlands J T M van der Meer D Paydafar

Laboratory of Pathology, Academic Medical Centre,

University of Amsterdam, Amsterdam, Netherlands F J W ten Kate

Laboratory of Medical Microbiology, Department of Clinical Virology, Academic Medical Centre,

University of

Amsterdam, Amsterdam, Netherlands P M E Wertheim-van Dillen

Department of Ophthalmo-Immunology, the Netherlands Ophthalmic Research Institute, Amsterdam, Netherlands A Kijlstra

Correspondence to: F D Verbraak, MD, Academic Medical Centre, University of Amsterdam, Department of Ophthalmology, PO Box 22700, 1100 DE

Amsterdam, Netherlands

Accepted for publication 29 January 1998

\begin{abstract}
Aim-To describe the risk of developing cytomegalovirus (CMV) retinitis after a first episode of extraocular CMV disease in AIDS patients.

Methods-A review of the clinical records of 20 AIDS patients, without CMV retinitis, with histologically confirmed extraocular CMV disease, was performed. The main outcome measures were occurrence of $\mathrm{CMV}$ retinitis, time to development of CMV retinitis, relation to maintenance therapy, and survival.

Results-A CMV retinitis was diagnosed in 17 of $20(85 \%)$ patients with an immunohistologically confirmed diagnosis of extraocular CMV disease after a mean follow up of 6.4 months. Four patients received maintenance therapy. Three of them developed retinitis after a mean of 9.6 months (range 2-16 months). Sixteen did not receive maintenance and retinitis was diagnosed in 14 of them after a mean of 5.7 months (range 2-11 months). Mean survival was 9.9 months after the diagnosis of extraocular disease, and 4.5 months after the diagnosis of retinitis. In the four patients receiving maintenance therapy, mean survival was 11.5 months, and in the 16 other patients mean survival was 9.5 months. Patients did not receive protease inhibitors.

Conclusion-In the preprotease inhibitor era extraocular CMV disease strongly predisposes to the subsequent development of CMV retinitis. Although maintenance therapy did not prevent the occurrence of retinitis, the time period between both events seems to lengthen considerably. In patients receiving maintenance survival is also longer.

(Br F Ophthalmol 1998;82:748-750)
\end{abstract}

Clinically manifest cytomegalovirus (CMV) disease is the most often diagnosed opportunistic viral infection in HIV positive patients. The annual incidence in patients with CD4+ lymphocyte counts below 100 cells $\times 10^{6} / 1$ is $14 \%$; and if CD4+ cell counts fall below 50 the incidence rises to $24 \%$ per year. The most debilitating, clinically significant CMV disease affects the eye, and CMV retinitis is present in approximately $90 \%$ of all cases with CMV disease. ${ }^{1-3}$

Extraocular CMV disease has been considered to predispose for developing CMV retini- tis, but exact data are not well known. Although maintenance therapy in case of CMV retinitis is mandatory, it is more questionable after other end organ disease. Most authors advise maintenance therapy only after a relapse of CMV disease. ${ }^{45}$ One study reported an equal number of newly diagnosed CMV retinitis with or without maintenance therapy after a first episode of gastrointestinal CMV disease. ${ }^{6}$ Even though new treatment modalities, such as oral ganciclovir, are now available, secondary prophylaxis is not routinely prescribed.

To answer the question, how often CMV retinitis occurs after an extraocular CMV disease, and whether there is a rationale for maintenance treatment after CMV end organ disease outside the eye, we carried out a retrospective analysis in all HIV positive patients seen in the AIDS department, who had a biopsy proved non-ocular CMV disease between March 1989 and March 1995. The incidence of CMV retinitis after a non-ocular CMV end organ disease and the time period between both events was registered, as was the survival after the occurrence of CMV disease. Additionally, the influence of maintenance therapy on these events was analysed.

\section{Patients and methods}

Medical records of patients admitted to the AIDS unit of the Academic Medical Centre between March 1989 and March 1995 with a biopsy proved first episode of extraocular CMV disease were reviewed.

For histopathology, biopsies were fixed in $10 \%$ buffered formalin ( $\mathrm{pH} 7.4$ ) and embedded in paraffin, processed, and routinely stained with haematoxylin and eosin. Sections $4 \mu \mathrm{m}$ thick were used for immunohistochemical detection of $\mathrm{CMV}$, using a monoclonal antibody against the immediate early antigen of CMV (E13, Biosoft Lab) with the streptavidin method. Before incubation, the slides were deparaffinised and pretreated with pepsin $0.25 \%$ in $0.01 \mathrm{HCl}$ for 10 minutes. Slides were read positive when nuclei of epithelial, endothelial, or stromal cells stained brightly with the antibody, whether or not nuclear inclusions were seen on the haematoxylin and eosin slides.

According to standard practice of the Academic Medical Centre all these patients were seen by an ophthalmologist at the time of diagnosis of extraocular CMV disease. Patients were included in this study if no retinitis was diagnosed at that time. All patients had to 
Table 1 Characteristics of 20 AIDS patients with a histopathologically confirmed first episode of extraocular CMV disease, but not yet suffering from retinitis

\begin{tabular}{|c|c|c|c|c|c|c|c|}
\hline \multirow[b]{2}{*}{ Patient no } & \multicolumn{2}{|c|}{ CMV extraocular } & \multirow[b]{2}{*}{$C D 4+$} & \multirow[b]{2}{*}{ Induction therapy } & \multirow[b]{2}{*}{ Maintenance } & \multirow{2}{*}{$\begin{array}{l}\text { Interval } \\
\text { E-Oc/Ret }\end{array}$} & \multirow[b]{2}{*}{ Survival } \\
\hline & Date & Localisation & & & & & \\
\hline 1 & $01-06-91$ & Upper GI & 20 & $\operatorname{GCV}(2) / \operatorname{Fosc}(1) / \mathrm{GCV}+\mathrm{Fosc}(2)$ & GCV & 16 & 8 \\
\hline 2 & 01-03-90 & Poly rad & 10 & $\mathrm{GCV}(1) / \mathrm{GCV}+\operatorname{Fosc}(2)$ & GCV & 11 & 2 \\
\hline 3 & $10-07-91$ & Upper GI & 40 & $\mathrm{GCV}(4)$ & GCV & - & 4 \\
\hline 4 & 07-04-89 & Upper GI & 10 & $\operatorname{GCV}(2) / F o s c(2)$ & Fosc & 2 & 3 \\
\hline 5 & $01-05-89$ & Lower GI & 10 & GCV(3) & - & 3 & 2 \\
\hline 6 & 01-07-92 & Lungs & 10 & GCV(3) & - & 2 & 2 \\
\hline 7 & 15-03-95 & Upper GI & 10 & GCV(2) & - & 4 & 4 \\
\hline 8 & $01-06-92$ & Upper GI & 10 & $\mathrm{GCV}(4)$ & - & 5 & 1 \\
\hline 9 & $15-09-92$ & Upper GI & 10 & GCV(2) & - & 2 & 2 \\
\hline 10 & $09-10-92$ & Upper GI & 10 & $\operatorname{GCV}(2) / \operatorname{Fosc}(2)$ & - & 6 & 6 \\
\hline 11 & $01-06-90$ & Upper GI & 40 & $\mathrm{GCV}(2)$ & - & 6 & 7 \\
\hline 12 & $20-01-94$ & Lower GI & 40 & GCV(3) & - & 9 & 3 \\
\hline 13 & $01-04-93$ & Lower GI & 10 & GCV(5) & - & 8 & 4 \\
\hline 14 & $08-05-95$ & Lower GI & 20 & Fosc (3) & - & 7 & 2 \\
\hline 15 & $09-12-93$ & Lower GI & 10 & $\mathrm{GCV}(2)$ & - & 11 & 5 \\
\hline 16 & $20-08-92$ & Lower GI & 10 & $\operatorname{GCV}(3) / \operatorname{Fosc}(1)$ & - & 9 & 12 \\
\hline 17 & $01-08-92$ & Lower GI & 20 & GCV(3) & - & 2 & 8 \\
\hline 18 & $01-02-91$ & Lower GI & 20 & $\mathrm{GCV}(2)$ & - & 6 & 1 \\
\hline 19 & $05-03-92$ & Lungs & 10 & $\operatorname{GCV}(1) / \operatorname{Fosc}(2)$ & - & - & 2 \\
\hline 20 & $20-09-90$ & Upper GI & 80 & $\mathrm{GCV}(2)$ & - & - & 11 \\
\hline
\end{tabular}

Date=date of diagnosis of extraocular CMV disease. Localisation: upper GI=upper gastrointestinal tract; lower GI=lower gastrointestinal tract; Lungs=CMV pneumonitis; Poly rad=CMV polyradiculopathy; CD4+=CD4 positive lymphocyte count, cells $\times 10^{6} / 1$. Induction therapy: GCV=ganciclovir $5 \mathrm{mg} / \mathrm{kg} / \mathrm{twice}$ daily; Fosc=foscavir $90 \mathrm{mg} / \mathrm{kg} / \mathrm{twice}$ daily; GCV+ Fosc=combination of both therapies. $(\mathrm{n})=$ number of weeks treated. Maintenance: $\mathrm{GCV}=$ ganciclovir $5 \mathrm{mg} / \mathrm{kg} / \mathrm{day} ; \mathrm{Fosc}=$ foscavir $90 \mathrm{mg} / \mathrm{kg} / \mathrm{day}$. Interval E-Oc/ Ret=time between extraocular CMV disease and CMV retinitis in months; $(-)=$ no CMV retinitis occurred. Survival=survival time after diagnosis of CMV retinitis in months.

respond favourably to induction therapy with either ganciclovir, $5 \mathrm{mg} / \mathrm{kg} /$ day twice daily, or foscavir, $90 \mathrm{mg} \mathrm{kg}$ /day twice daily, or a combination of both drugs. Maintenance therapy after extraocular CMV disease is generally not prescribed. All patients included had regular, monthly eye examinations by an ophthalmologist after the occurrence of an extraocular CMV disease. According to the CDC classification, CMV retinitis was diagnosed when a necrotising retinitis with a characteristic "cheese-like" appearance was present with or without haemorrhages. ${ }^{8}$ Additionally a favourable response to therapy had to be present.

Entry criteria were fulfilled in 20 patients. Patient characteristics are shown in Table 1. Mean age at diagnosis of extraocular CMV disease was 42 years (range $32-58$ years). In two patients the CMV disease was the AIDS defining diagnosis. In the other 18 patients the AIDS diagnosis preceded the diagnosis of CMV disease with a mean of 2 years (range $0.5-8$ years). AIDS defining diagnosis was seven times Pneumocystis carinii pneumonitis, five times Kaposi's sarcoma, four times candida oesophagitis, and twice cryptosporidial diarrhoea.

For statistical analysis, comparing the time period between the first non-ocular CMV disease and the occurrence of CMV retinitis, in the patients receiving maintenance therapy versus the patients not receiving maintenance, the Kaplan-Meier method and the log rank test were used.

\section{Results}

In 17 of $20(85 \%)$ patients with an immunohistologically proved non-ocular clinically manifest CMV infection a CMV retinitis occurred after a mean follow up of 6.4 months (range 2-16 months, see Table 1). Four patients (patients 1-4, Table 1) received maintenance therapy, $5 \mathrm{mg}$ ganciclovir/kg/day or $90 \mathrm{mg}$ foscavir/ $\mathrm{kg} / \mathrm{day}$, and in three of them CMV retinitis was diagnosed after 2, 11 , and 16 months (mean 9.6 months). The fourth patient (patient 3) died after a follow up of 4 months without the occurrence of retinitis. In one patient (patient 4) maintenance therapy was stopped after 1 month because of drug toxicity, 1 month before a diagnosis of retinitis. Sixteen patients did not receive maintenance. In 14 of these patients $(87.5 \%)$ a CMV retinitis was diagnosed after a mean follow up of 5.7 months, range 2-11 months. Two patients (patients 19 and 20) died without the occurrence of retinitis, after 2 and 11 months respectively. Statistical analysis showed that the difference in the time between both events was not statistically significant $\left(\chi^{2}=1.8545, \mathrm{p}=\right.$ $0.17)$.

Mean survival after a diagnosis of nonocular CMV disease was 11.5 months in the four patients with maintenance and 9.5 months in the 16 patients without maintenance. Mean survival after a diagnosis of CMV retinitis was 4 months in the maintenance group and 4.5 months in the non-maintenance group of patients.

\section{Discussion}

This study shows that extraocular CMV disease is a major risk factor for developing CMV retinitis. Seventeen out of 20 (85\%) AIDS patients with a biopsy proved extraocular CMV disease developed CMV retinitis after a mean follow up of 6.4 months.

Although numbers are small, maintenance therapy seems to postpone the development of CMV retinitis after an extraocular CMV disease. Without maintenance therapy retinitis occurred after a mean follow up of 5.7 months, whereas with maintenance therapy the mean retinitis-free interval became 9.6 months (2, 11 , and 16 months). Owing to the relatively 
small number of patients this difference did not reach statistical significance.

One recent study reported CMV retinitis to occur in $22 \%$ of 239 HIV positive patients within 1 year of follow up. CD4+ lymphocyte count was less than 50 cells $\times 10^{6} / 1$ in over $90 \%$ of these patients. ${ }^{2}$ In another prospective study of $367 \mathrm{HIV}$ positive patients, with a CD4+ count below 100 cells $\times 10^{6} / 1$, and a mean follow up of 2.9 years, $32 \%$ of the patients developed a CMV retinitis.

Both patient groups described are comparable with the patients included in this study. All patients have the same disease stage and equal CD4 positive lymphocyte counts. Comparing the incidence of CMV retinitis in the patients included in this study, $85 \%$ in 2 years, with the reported incidence in the literature, $22 \%$ in 1 year and $35 \%$ in 2.9 years, proves extraocular CMV disease to be a major risk factor for developing subsequent retinitis.

To the best of our knowledge there are no reports that systematically look for retinitis as a secondary event after a first episode of extraocular CMV disease or the effect of maintenance therapy in these cases. The overall conclusion out of those studies at least mentioning the occurrence of CMV retinitis after a first episode of extraocular CMV disease is that the association between both events is very high. ${ }^{39-13}$

In a study describing the natural history and necropsy findings in a cohort of 1227 HIV positive patients, seen between 1984 and 1994, the risk of a relapse of CMV disease was significantly higher in patients not receiving maintenance therapy. ${ }^{14}$ Moreover, relapses occurred later in patients given maintenance therapy compared with those without maintenance treatment $(20 \%$ of patients, median time of 17 months, versus $62 \%$, median time of 5.5 months). Maintenance therapy however did not improve survival, with a mean of 8 months in both groups. Relapse was defined in this study as a new CMV organ disease at the same or different site after a complete resolution of the first episode. No details were provided concerning the occurrence of CMV retinitis after a diagnosis of extraocular CMV disease.

A previous study however claims no effect of maintenance therapy after a successful treatment of gastrointestinal CMV disease. ${ }^{6} \mathrm{Nei}-$ ther the number of relapses of gastrointestinal CMV disease, nor the number of CMV retinitis developing, nor the median time to occurrence of this second event differed between patients receiving maintenance therapy or patients without maintenance therapy. In the latter study, the decision to embark on further treatment was left to the clinical investigator unless there was concurrent retinitis; in such cases, maintenance therapy was started routinely. The maintenance receiving group must have included patients with retinitis from the start and this must have influenced the recurrence rate in a negative way, in light of the high rate of recurrence of $\mathrm{CMV}$ retinitis despite maintenance therapy.
Protease inhibitors were not generally prescribed during the time period the patients included in this study were seen, and in fact there were no patients included who received protease inhibitors.

Considering the $85 \%$ of patients with a diagnosis of CMV retinitis, following a first episode of extraocular CMV disease, after a mean follow up of 6.4 months, found in this study, it seems obvious that extraocular CMV disease strongly predisposes to the subsequent development of CMV retinitis. CMV retinitis occurred despite the fact that extraocular CMV disease seemed to be completely healed after 3-5 weeks of antiviral treatment. Although maintenance treatment did not prevent the occurrence of CMV retinitis the time interval between both events was considerably longer in patients receiving maintenance therapy. One patient receiving maintenance therapy with foscavir, $90 \mathrm{mg} / \mathrm{kg} /$ day, had to stop, owing to drug toxicity. This patient developed CMV retinitis within 1 month after stopping the maintenance therapy. This patient is a good example of both the desirability of an effective maintenance therapy and the unwanted toxic side effects of the drugs available today.

Although our study does not provide conclusive evidence in favour of maintenance therapy after an initial extraocular CMV disease, frequent ophthalmic examinations are definitely warranted in such patients. The introduction of protease inhibitors and the use of anti-HIV multidrug combination therapy may alter the treatment strategies against CMV drastically in the near future.

We would like to thank Professor Marc D de Smet for critically reading the manuscript.

1 Gallant JL, Moore RD, Richman DD, et al. Incidence and natural history of cytomegalovirus disease in patients with natural history of cytomegalovirus disease in patients with with zidovudine. F Infect Dis 1992;166:1223-7.

2 Spector SA, McKinley GF, Lalezar JP, et al. Oral ganciclovir for the prevention of cytomegalovirus disease in persons with AIDS. N Engl f Med 1996;334:1491-7.

3 Hoover DR, Peng Y, Saah A, et al. Occurrence of cytomegalovirus retinitis after human immunodeficiency virus immunosuppresion. Arch Ophthalmol 1996;114:821-7.

4 Chui Dw, Owen RL. AIDS and the gut. $f$ Gastroenterol Hepatol 1994;9:291-303.

5 Sutton FM, Graham DY, Goodgame RW. Infectious esophagitis. Gastrointest Endosc Clin NA 1994;4:713-29.

6 Blanshard C, Benhamon Y, Dohin E, et al. Treatment of AIDS-associated gastrointestinal cytomegalovirus infection with foscarnet and ganciclovir: a randomized comparison.

f Infect Dis 1995;172:622-8.
7 Danner SA. Management of cytomegalovirus disease. AIDS 195;9(Supp1 2):S3-8.

8 Centers for Disease Control. Revision of the CDC surveillance case definition for acquired immunodeficiency surveillance case definition for acquired in
syndrome. MMWR Suppl 1987;36:1S-3S.

9 Salomon N, Gomez T, Perlman DC, et al. Clinical features and outcome of HIV-related cytomegalovirus pneumonia. AIDS 1997;11:319-24.

10 van der Meer JTM, Drew WL, Bowden RA, et al. Summary of the international consensus symposium on advances on the diagnosis, treatment and prophylaxis of cytomegalovirus infection. Antiviral Res 1996;32:119-40.

1 Mentec O, Leport C, Leport J, et al. Cytomegalovirus colitis in HIV-1-infected patients: a prospective research in 55 patients. AIDS 1994;8:461-7.

12 Dieterich DT, Kotler DP, Busch DF, et al. Ganciclovir treatment of cytomegalovirus colitis in AIDS: a randomized double-blind, placebo-controlled multicenter study. F Infect Dis 1993;167:278-82.

13 Dieterich DT, Rahmin M. Cytomegalovirus colitis in AIDS: presentation in 44 patients and a review of the literature. $\mathscr{f}$ presentation in 44 patients and a review of the literat

14 d'Arminio Montforte A, Mainini F, et al. Predictors of cytomegalovirus disease, natural history and autopsy findings in a cohort of patients with AIDS. AIDS 1997;11:51724 . 